\title{
Sheep meat polyunsaturated fatty acids and human health in relation to recent nutritional guidelines
}

\author{
E. Kasapidou and P. Mitliaga \\ School of Agricultural Technology. Technological Educational Institute of West Macedonia, Florina, Greece
}

Sheep meat has a high content of saturated fatty acids (SFA) and a low content in polyunsaturated fatty acids (PUFA) raising concerns on the nutritive value of the consumed meat. The link between SFA and PUFA intake, and cardiovascular disease has been well documented in a plethora of studies ${ }^{(1)}$. Reductions on the intake of SFA and increases on the intake of long chain $n$ - 3 PUFA and particularly eicosapentaenoic (EPA) and docosahexaenoic (DHA) are recommended for the reduction of cardiovascular disease risk. Attention has been focused on the production of sheep meat with a beneficial fatty acid composition promoting cardiovascular health. The objective of this work is to examine the fatty acid composition of lean sheep meat in relation to the recent dietary recommendations for healthy intake of dietary fatty acids.

Scopus, a database of peer-reviewed research literature was searched for publications referring to fatty acid composition in sheep meat using the default search field "Title-Abstract-Keywords" excluding review and conference papers from the search. Research was narrowed in the decade 2002-2012 to encompass modern production trends in animal nutrition. Intramuscular fatty acid composition was examined for (i) the SFA and PUFA proportions in relation to partial replacement of SFA with PUFA and (ii) the EPA and EPA content. In the end 19 reports that the reported data met either one or both selection criteria and allowed for necessary additional recalculations were included in the study. SFA and PUFA proportions were calculated as reported by Raes et $a l^{(3)}$ for uniform comparison between the different studies.

Data from 10 studies showed that an increase in the proportion of PUFA and parallel decreases in the SFA proportion were achieved in only 4 studies. In the other studies, there was no consistent pattern for the substitution of SFA with PUFA. Effective substitution of SFA with PUFA was particularly low and inadequate to alter the nutritional quality of meat. Partial replacement of SFA and PUFA was mainly achieved by alterations in the lipid content and fatty acid composition of animal diets. Most effective changes were associated with high forage diets that offer both economical advantages and environmental benefits in traditional and alternative feeding systems. Reported data in 13 studies deriving from 45 different experimental treatments showed that the average content of EPA + DHA was $46 \mathrm{mg} / 100 \mathrm{~g}$ of meat whereas the range lies from as low as 9 up to $136 \mathrm{mg} / 100 \mathrm{~g}$ of meat. These results show that a serving of sheep meat has a small contribution to the overall recommended intake of $250 \mathrm{mg} / \mathrm{d}$ for EPA $+\mathrm{DHA}^{(2)}$. Intramuscular fat content as well as animal diet are primarily related to the content of EPA + DHA in sheep meat. Nutritional and to lesser extent genetic approaches have been employed to modify the fatty acid composition of sheep meat. Other factors such as breed, rearing system, slaughter age and weight, and muscle type affect fatty composition making the interpretation of the results difficult. Therefore, this work aimed to provide an insight of the potential changes in fatty acid composition of sheep meat as reported in recent studies. Factors such as intramuscular fat content and fatty acid profile of each lipid class are also important parameters for the healthiness of sheep meat.

Fatty acid composition of sheep meat is not constant and it can be altered by animal nutrition ${ }^{(4)}$. Further research is needed for the production of sheep meat with a favorable composition for disease prevention according to the latest nutritional guidelines.

1. Salter AM (2012) Animal 11, 1-9.

2. European Food Safety Authority (2009) EFSA J 1176, 1-11.

3. Raes K, De Smet, S \& Demeyer (2004) Anim Feed Sci Technol 113, 199-221.

4. Givens I (2009) Nutrients 1, 71-82. 